\title{
Multipartite Entanglement in Stabilizer Tensor Networks
}

\author{
Sepehr Nezami ${ }^{1,2}$ and Michael Walter ${ }^{1,3}$ \\ ${ }^{1}$ Stanford Institute for Theoretical Physics, Stanford University, Stanford, California 94305, USA \\ ${ }^{2}$ Institute for Quantum Information and Matter and Walter Burke Institute for Theoretical Physics, \\ California Institute of Technology, Pasadena, California 91125, USA \\ ${ }^{3}$ Korteweg-de Vries Institute for Mathematics, Institute for Theoretical Physics, \\ Institute for Logic, Language, and Computation, and QuSoft, University of Amsterdam, 1098 XG Amsterdam, Netherlands
}

(Received 21 September 2020; accepted 11 November 2020; published 10 December 2020)

\begin{abstract}
Despite the fundamental importance of quantum entanglement in many-body systems, our understanding is mostly limited to bipartite situations. Indeed, even defining appropriate notions of multipartite entanglement is a significant challenge for general quantum systems. In this work, we initiate the study of multipartite entanglement in a rich, yet tractable class of quantum states called stabilizer tensor networks. We demonstrate that, for generic stabilizer tensor networks, the geometry of the tensor network informs the multipartite entanglement structure of the state. In particular, we show that the average number of Greenberger-Horne-Zeilinger (GHZ) triples that can be extracted from a stabilizer tensor network is small, implying that tripartite entanglement is scarce. This, in turn, restricts the higher-partite entanglement structure of the states. Recent research in quantum gravity found that stabilizer tensor networks reproduce important structural features of the AdS/CFT correspondence, including the Ryu-Takayanagi formula for the entanglement entropy and certain quantum error correction properties. Our results imply a new operational interpretation of the monogamy of the Ryu-Takayanagi mutual information and an entropic diagnostic for higher-partite entanglement. Our technical contributions include a spin model for evaluating the average GHZ content of stabilizer tensor networks, as well as a novel formula for the third moment of random stabilizer states, which we expect to find further applications in quantum information.
\end{abstract}

DOI: 10.1103/PhysRevLett.125.241602

Introduction.-Quantum entanglement is of fundamental relevance for the behavior of quantum mechanical systems in condensed matter and high energy physics. From the perspective of quantum information processing, it is the resource that provides speedups in quantum computing, security in quantum cryptography, and improved performance in quantum sensing. However, the structure of many-body or multipartite entanglement is only poorly understood [1]. In this work, we focus on analyzing multipartite entanglement in an important but tractable class of quantum states known as stabilizer tensor networks, i.e., tensor networks that are obtained by contracting stabilizer states. Stabilizer states are an important family of quantum states that can be highly entangled (even maximally so) but still have sufficient algebraic structure to admit an efficient classical description. This makes them a versatile tool in quantum information theory, particularly in the theory of quantum error correction [2]. Of particular import in the present context is that the tripartite entanglement structure of stabilizer states can be precisely quantified - any tripartite stabilizer state is locally equivalent to a collection of bipartite Bell pairs and tripartite GHZ states [3,4] (cf. Refs. [5-11]).

An important additional motivation to study stabilizer tensor networks comes from current research in quantum gravity. In recent years, research in quantum gravity and quantum information theory has been inspired by a fruitful mutual exchange of ideas. Tensor networks in particular provide a common framework, rooted in the similarity between the structure of the tensor network and the bulk geometry in holographic duality [12-14]. A paradigmatic example is the Ryu-Takayanagui formula, $S(A) \simeq\left|\gamma_{A}\right| / 4 G_{N}$, which asserts that the entanglement entropy of a boundary region $A$ in a holographic state is in leading order proportional to the area of a corresponding minimal surface $\gamma_{A}$ in the bulk geometry $[15,16]$. Likewise, in any tensor network, the entanglement entropy of a boundary subsystem can be upper-bounded in terms of the size of a minimal cut through the network [17] (Fig. 1). This bound can be saturated not only through the choice of suitable tensors $[18,19]$ but is in fact a generic phenomenon in random tensor networks with large bond dimension $[20,21]$, the mechanism of which can be understood in terms of multipartite entanglement distillation. These tensor network models not only reproduce the Ryu-Takayanagi formula for the entanglement entropy, but they also implement several other significant features of holographic duality [18-20]. In many ways, these properties follow from the bipartite entanglement structure and can be therefore reduced to entropic considerations. 


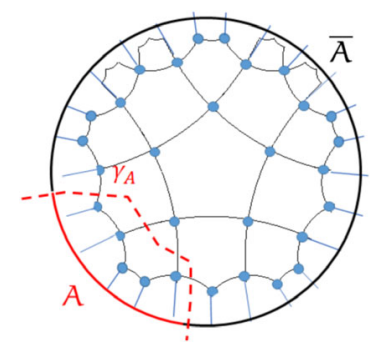

FIG. 1. Stabilizer tensor networks. A tensor network state is obtained by placing random stabilizer states at the bulk vertices (blue) and contracting according to the edges of the graph. In the limit of large bond dimensions, the average entanglement entropy of a boundary region $A$ is proportional to the length of a minimal cut $\gamma_{A}$ through the network (dashed line) [20], $S(A) \simeq S_{\mathrm{RT}}(A)$, reproducing the Ryu-Takayanagi formula in holography.

In this Letter, we initiate a study of multipartite entanglement in random tensor network models. Our motivation is twofold: First, recent research in quantum gravity has raised profound questions regarding the multipartite entanglement in holographic states [22-24], in particular with regards to tripartite entanglement of GHZ type $[25,26]$.

Answers to these questions in the context of tensor network models will likely lead to new diagnostics applicable in holography. Second, we seek to understand the general mechanisms by which quantum information is encoded in tensor networks; an improved understanding of the entanglement structure may inform the design of tensor networks that adequately represent the physics. While it is possible to obtain partial information from the entanglement entropy of subsystems [22,23,27-29], many basic questions regarding the multipartite entanglement cannot be answered from entropic data. A striking example is that a pair of GHZ states cannot be entropically distinguished from three Bell pairs, even though their entanglement properties are vastly different [30].

Summary of results. Our main result is that the average amount of tripartite entanglement in random stabilizer networks is small. More precisely, for any tripartition the expected number of GHZ triples remains bounded as we take the limit of large bond dimensions (Theorem 1). This has a number of surprising consequences on the correlation and entanglement structure: (a) The number of Bell pairs that can be extracted between two subsystems $A$ and $B$ is roughly half the mutual information $I(A: B)$ (which in turn can be read off the geometry of the network using the Ryu-Takayanagi formula); (b) in particular, the mutual information measures quantum entanglement, proving a conjecture in Ref. [22] for stabilizer tensor networks; (c) the monogamy of the mutual information, $I(A: B)+I(A: C) \leq I(A: B C)$, established in Ref. [22] for holographic entropies, thus acquires an operational interpretation as originating from the monogamy of quantum entanglement; (d) the tripartite information $I_{3}:=I(A: B)+$ $I(A: C)-I(A: B C)$ (i.e., the difference in the above
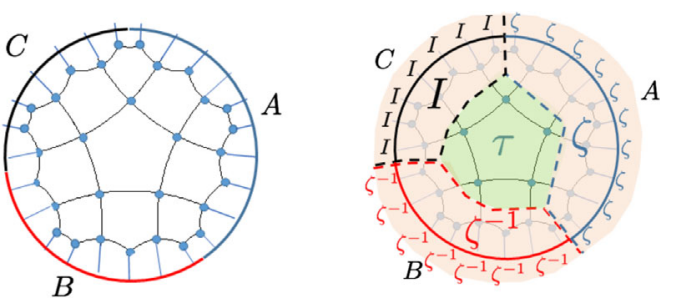

FIG. 2. Tripartite entanglement and the GHZ spin model. Left. Tripartition of the boundary. Right. Illustration of the spin model (with boundary conditions and minimal energy configuration) used to evaluate the $\mathrm{GHZ}$ content of a random stabilizer tensor network state.

inequality) provides a diagnostic for four-partite entanglement; in fact, after extracting all Bell pairs we obtain a residual four-partite entangled state with the entropies of a perfect tensor of size $-I_{3} / 2$ [18], strengthening the picture provided by the holographic entropy cone [28] (Fig. 3).

We establish these results based on two main technical contributions: First, we diagnose the GHZ content by a polynomial invariant (the third moment of the partial transpose $\rho_{A B}^{T_{B}}$ ). Its average can be evaluated using a classical ferromagnetic spin model, the GHZ spin model. For large bond dimensions, this model is in its lowtemperature (ordered) phase and hence the tripartite entanglement is determined by its minimal energy configurations (Fig. 2). Second, we derive a novel formula for the third moment of nonqubit stabilizer states. It refines the results of Refs. [31-33] and we expect that it will be of similar interest in quantum information theory. Throughout this Letter, we measure entropies of $p$-level systems in units of $\log _{p}$ bits.

Random stabilizer networks.-We now describe the random stabilizer network model. Consider a connected graph with vertices $V$ and edges $E$ (parallel edges allowed). Let $V_{\partial}$ denote a subset of the vertices, which we will refer to as the boundary vertices; all other vertices are called bulk vertices and denoted by $V_{b}$. Given a choice of bond
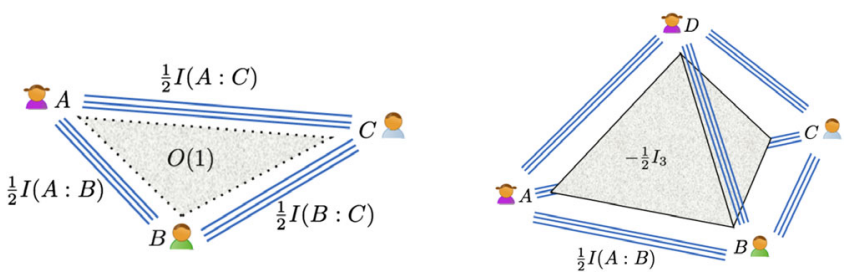

FIG. 3. Multipartite entanglement structure. Left. For any tripartition, there is only a bounded number of GHZ triples (dashed triangle) and hence the entanglement is dominated by bipartite maximal entanglement (blue lines). Right. For four (and more) parties, we can likewise extract maximally entangled pairs between any two parties (blue lines). The residual state has approximately the entropies of a perfect tensor (tetrahedron). This decomposition is in one-to-one correspondence with the extreme rays of the holographic entropy cone [28]. 
dimensions for all edges, we define a pure quantum state by placing tensors $\left|V_{x}\right\rangle$ at the bulk vertices and contracting according to the edges:

$$
|\Psi\rangle=\left(\underset{x \in V_{b}}{\otimes}\left\langle V_{x}\right|\right)(\underset{e \in E}{\otimes}|e\rangle) .
$$

Here, $|e\rangle \propto \sum_{i}|i i\rangle$ denotes a normalized maximally entangled state corresponding to an edge $e$. The state $|\Psi\rangle$ is a tensor network state defined on the Hilbert space corresponding to the boundary vertices $V_{\partial}$, and in general unnormalized. We write $\rho=\Psi / \operatorname{tr} \Psi$ for the normalized density matrix, where $\Psi=|\Psi\rangle\langle\Psi|$. See Fig. 1 for an illustration.

To build a stabilizer tensor network state, we choose bond dimensions of the form $D=p^{N}$, where $p$ is a fixed prime and $N$ some positive integer that we will later choose to be large (for simplicity of exposition, we choose all bond dimensions to be the same). Thus the Hilbert space associated with a single vertex is of dimension $D_{x}=p^{N \operatorname{deg}(x)}$, where $\operatorname{deg}(x)$ denotes the degree of the vertex (i.e., the number of incident edges), and the Hilbert spaces associated with the bulk vertices has dimension $D_{b}=p^{N_{b}}$, where $N_{b}=N \sum_{x \in V_{b}} \operatorname{deg}(x)$. We now select each vertex tensor $V_{x}$ in Eq. (1) independently and uniformly at random from the set of stabilizer states. Thus $\Psi$ is obtained by partially projecting one stabilizer state onto another (viz., the random vertex tensors onto the maximally entangled pairs), which implies that either $\Psi$ is zero or again a stabilizer state. In the latter case, which occurs with high probability for large $N$, we say that $\Psi$ is a random stabilizer tensor network state. In any tensor network state, the entanglement entropy $S(A)=$ $-\operatorname{tr} \rho_{A} \log _{p} \rho_{A}$ of a boundary subsystem $A \subseteq V_{\partial}$ can always be upper bounded by $S_{\mathrm{RT}}(A):=N \min \left|\gamma_{A}\right|$ [34], where we minimize over all cuts $\gamma_{A}$ that separate the subsystem $A$ from its complement $\bar{A}$ in $V_{\partial}$ (Fig. 1). Formally, such a cut is defined by a subset of vertices $V_{A}$ that contains precisely those boundary vertices that are in $A$ such that the set of edges that leaves $V_{A}$ is $\gamma_{A}$.

The fundamental property of random tensor networks is that in the limit of large $N$ (or large $p$ ), this upper bound becomes saturated [20]. Thus these models reproduce the Ryu-Takayanagi formula in holography. More precisely, the average entanglement entropy of a boundary subsystem, conditioned on the tensor network state being nonzero, is given by

$$
\langle S(A)\rangle_{\neq 0} \simeq S_{\mathrm{RT}}(A) .
$$

Here and in the following, we write $\simeq$ for equality up to order $O(1)$, independent of $N$. The central fact used to derive this is that random stabilizer states form a projective 2-design [35,36], i.e., that their first and second moments agree with the Haar measure. For the reader's convenience, and since the derivation in Ref. [20] focused on the case of large $p$, we give a succinct derivation in the Supplemental Material [37]. This result can be strengthened to show that in fact $S(A) \simeq S_{\mathrm{RT}}(A)$ with high probability [20].

Tripartite entanglement.-Any pure tripartite stabilizer state $\rho_{A B C}$ is locally equivalent to a tensor product of bipartite maximally entangled states, $\left|\Phi^{+}\right\rangle_{A B} \propto \sum_{i=1}^{p}|i i\rangle$, etc., and tripartite GHZ states $|\mathrm{GHZ}\rangle_{A B C} \propto \sum_{i=1}^{p}|i i i\rangle$ $[3,4]$. That is, there exists a local unitary $U=U_{A} \otimes U_{B} \otimes$ $U_{C}$ such that $U \rho_{A B C} U^{\dagger}$ is equal to

$$
\left(\Phi_{A B}^{+}\right)^{\otimes c} \otimes\left(\Phi_{A C}^{+}\right)^{\otimes b} \otimes\left(\Phi_{B C}^{+}\right)^{\otimes a} \otimes \mathrm{GHZ}_{A B C}^{\otimes g}
$$

(we suppress local states on $A, B$ and $C$ which do not impact the entanglement). The integers $a, b, c, g \geq 0$ are uniquely determined; thus they meaningfully characterize the bipartite and tripartite entanglement between subsystems $A, B$, and $C$. Now, we can state our main result (The proof is postponed to Sec. V):

Theorem 1. (Tripartite entanglement in random stabilizer networks) Let $A, B, C$ denote a tripartition of the boundary (Fig. 2. left), and $p \equiv 2(\bmod 3)$. Then the expected number of GHZ states in a random stabilizer network is of order $O(1)$ in the limit of large $N$.

Explicitly, we have the following bound in terms of the geometry of the tensor network:

$$
\langle g\rangle_{\neq 0} \leq \#_{b} \log _{p}(p+1)+\log _{p}\left(\#_{A} \#_{B} \#_{C}\right)+4 \delta,
$$

with $\#_{A}$ the number of minimal cuts for $A$, etc., $\#_{b}$ the maximal number of components of any subgraph obtained by removing minimal cuts for $A, B C$ [39], and $\delta=(2 p+2)^{V_{b}} / p^{N}$.

In most cases of interest, the minimal cuts are unique and there remains a single connected component after their removal, so that $\langle g\rangle_{\neq 0} \leq \log _{p}(p+1)+4 \delta$ [40]. We note that Markov's inequality implies that the number of GHZ triples in fact remains bounded with high probability. Theorem 1 vastly generalizes the bound in Ref. [9], which can be obtained as the special case for a graph with a single bulk vertex.

In general, the mutual information is sensitive to both classical and quantum correlations. For a general stabilizer state of the form (3), $I(A: B)=2 c+g$, where $c$ is the number of maximally entangled pairs and $g$ the number of GHZ triples (whose reduced state on $\mathrm{AB}$ is a classically correlated state). In random stabilizer networks, however, Theorem 1 shows that $\langle g\rangle_{\neq 0}$ is bounded. Thus the average number of maximally entangled pairs that can be extracted between $A$ and $B$ is roughly one half the mutual information, $I(A: B) / 2 \simeq c$, which in turn can be estimated from the geometry of the tensor network by using the RyuTakayanagi formula (2). In particular, bipartite correlations between any two boundary subsystems are dominated by quantum entanglement and determined rigidly by the 
geometry of the tensor network, confirming a property that is also suspected to hold in holography [22] (Fig. 3. left).

Higher-partite entanglement.-Theorem 1 has a number of remarkable consequences for the entanglement structure for four and more subsystems. We first consider the extraction of bipartite entanglement. Consider a random stabilizer tensor network state whose boundary is partitioned into $k$ subsystems $A_{1}, A_{2}, \ldots, A_{k}$. Applying the preceding discussion to $A=A_{i}, B=A_{j}$ and $C=A_{i} A_{j}$ their complement, we find that the average number of maximally entangled pairs that can be extracted between any two subsystems $A_{i}$ and $A_{j}$ is $t_{i j} \simeq I\left(A_{i}: A_{j}\right) / 2$. The extraction process is implemented by local unitaries $U_{i} \otimes U_{j}$; it leaves all other mutual informations invariant and does not introduce new GHZ triples. We can therefore repeat the process and extract maximally entangled pairs between any pair of subsystems $A_{i}$ and $A_{j}$, until we obtain a residual state $\tilde{\rho}_{A_{1} \ldots A_{k}}$ whose bipartite mutual informations $I\left(A_{i}: A_{j}\right)$ is all of order $O(1)$.

We now specialize the preceding discussion to a fourpartite system $(k=4)$. Here, the vanishing of the pairwise mutual informations implies that the entropies of the residual state will have the following simple form: $S\left(A_{i}\right) \simeq$ $\frac{1}{2} S\left(A_{i} A_{j}\right) \simeq m$ for all $i \neq j$, where $m \geq 0$ is some integer [28]. Ignoring the order-one corrections, stabilizer states with such entropies are four-partite perfect tensors. These are tensors that are unitaries from any pair of subsystems to the complement, a crucial property used in the explicit construction of holographic codes $[18,19]$. Significantly, it is possible to determine $m$ from the entropies of the original state, or, more specifically, from its tripartite information $I_{3}:=I\left(A_{1}: A_{2}\right)+I\left(A_{1}: A_{3}\right)-I\left(A_{1}: A_{2} A_{3}\right)$, which is invariant under the extraction of the maximally entangled pairs (it also does not depend on the choice of $A_{1}, A_{2}, A_{3}$ ). In short, we have established the following result:

Theorem 2. (Four-partite entanglement in random stabilizer networks) Let $A_{1}, \ldots, A_{4}$ denote a partition of the boundary into four subsystems. Then the random stabilizer network state is locally equivalent to

$$
\underset{i \neq j}{\otimes}\left(\Phi_{A_{i} A_{j}}^{+}\right)^{t_{i j}} \otimes \tilde{\rho}_{A_{1} A_{2} A_{3} A_{4}} .
$$

In the limit of large $N$, on average $t_{i j} \simeq \frac{1}{2} I\left(A_{i}: A_{j}\right)$ and the residual state $\tilde{\rho}$ has approximately the entropies of a perfect tensor of size $-I_{3} / 2$ [that is, $S\left(A_{i}\right) \simeq S\left(A_{i} A_{j}\right) / 2 \simeq-I_{3} / 2$ ].

Our result provides a new interpretation of the tripartite information $I_{3}$ for random stabilizer networks-namely, as a measure of the entropy of the residual, genuinely fourpartite entangled state $\tilde{\rho}$. Since entropies are always non-negative, it follows that $I_{3} \lesssim 0$; equivalently, the mutual information is monogamous, $I(A: B)+I(A: C) \lesssim$ $I(A: B C)$, as was proved for holographic entropies in Ref. [22]. This can also be seen by observing that, in our setting, one half the mutual information is an entanglement measure; it is up to $O(1)$ corrections equal to, e.g., the squashed entanglement $E_{\mathrm{sq}}$ [41]; therefore, the monogamy of the mutual information also follows as a direct consequence of the monogamy of the latter.

Lastly, we note that although many important physical states are stabilizers (such as the ground state of systems with commuting Pauli Hamiltonians, e.g., the toric code or several fracton models [42], and certain Chern-Simons theories [43]), most states are far from this ensemble. Therefore, one should exercise caution in generalizing our results to other scenarios.

Method: The GHZ spin model.-We now sketch the proof of Theorem 1. Previous works such as Ref. [9] have calculated the GHZ content of multiqubit stabilizer states by using the algebraic formula from Ref. [3] in terms of dimensions of colocal stabilizer subgroups. Here, we proceed differently. The idea is to use the partial transpose $\rho_{A B}^{T_{B}}$ of the reduced state, which is sensitive to bipartite entanglement. A short calculation using Eq. (3) shows that $\operatorname{tr}\left(\rho_{A B}^{T_{B}}\right)^{3}=p^{-2(a+b+c+g)}$. Thus the number of GHZ states contained in a tripartite stabilizer state can be computed as

$$
g=S(A)+S(B)+S(C)+\log _{p} \operatorname{tr}\left(\rho_{A B}^{T_{B}}\right)^{3} .
$$

In a random stabilizer network, we can upper bound $S(A) \leq S_{\mathrm{RT}}(A)$, etc., and we know from the preceding section that this bound is not too lose. The main challenge is to upper-bound the expectation value $\left\langle\operatorname{tr}\left(\Psi_{A B}^{T_{B}}\right)^{3}\right\rangle$, which is a third moment in the unnormalized random tensor network state (1). In general, it is well known that a mixed quantum state $\rho_{A B}$ has bipartite entanglement if $\rho_{A B}^{T_{B}}$ has negative eigenvalues, hence, moments of $\rho_{A B}^{T_{B}}$ should contain information about the multipartite entanglement of the global pure state [44]. This connection is particularly sharp for stabilizer states through Eq. (5), but we expect the similar calculations to be informative for other ensembles of quantum states.

We start with the multiqubit case $(p=2)$. Only in this case we can use the recent result that multiqubit stabilizers are projective 3-designs [32,33]. Thus we have that for each vertex tensor $\left\langle\mid V_{x}\right\rangle\left\langle\left. V_{x}\right|^{\otimes 3}\right\rangle=\sum_{\pi \in S_{3}} R_{x}(\pi) /$ $D_{x}\left(D_{x}+1\right)\left(D_{x}+2\right)$, where we sum over all permutations $\pi \in S_{3}$ and write $R_{x}(\pi)$ for the corresponding permutation operator acting on three copies of the vertex Hilbert space. Using the analogous notation, we find that $\operatorname{tr}\left(\Psi_{A B}^{T_{B}}\right)^{3}=\operatorname{tr} \Psi^{\otimes 3} R_{A}(\zeta) R_{B}\left(\zeta^{-1}\right)$, where $\zeta$ is the cyclic permutation that sends $1 \mapsto 2 \mapsto 3$. A careful calculation then reveals that

$$
\left\langle\operatorname{tr}\left(\Psi_{A B}^{T_{B}}\right)^{3}\right\rangle \leq 2^{-3 N_{b}} \sum_{\left\{\pi_{x}\right\}} 2^{-N \sum_{\langle x y\rangle} d\left(\pi_{x}, \pi_{y}\right)},
$$

where the sum is over all choices of permutations $\pi_{x} \in S_{3}$, subject to the boundary conditions $\pi_{x}=\zeta$ for $x \in A$, $\pi_{x}=\zeta^{-1}$ in $B$, and $\pi_{x}=1$ in $C$; the sum in the exponent is over all edges, and we define $d\left(\pi_{x}, \pi_{y}\right)$ as the minimal 
number of transpositions required to go from one permutation to the other. We can interpret the right-hand side of Eq. (6) as the partition sum of a ferromagnetic spin model with permutation degrees of freedom at each vertex at inverse temperature $\log N$ (Fig. 2, right).

For large $N$, we are in the low-temperature (ordered) phase and the partition function is dominated by the minimal energy configuration:

$$
\sum_{\left\{\pi_{x}\right\}} 2^{-N \sum_{\langle x y\rangle} d\left(\pi_{x}, \pi_{y}\right)} \leq 2^{-N E_{0}}(\#+\delta),
$$

where $E_{0}$ denotes the minimal energy, \# the number of minimal energy configurations, and $\delta=6^{V_{b}} / 2^{N}$. Now consider an arbitrary configuration $\left\{s_{x}\right\}$, minimal or not. If we denote by $V_{A}$ the $\zeta$ domain then the boundary conditions ensure that $V_{A}$ is a cut separating $A$ from $B C$. While this cut is not necessarily minimal, we always have that $N\left|\partial V_{A}\right| \geq S_{\mathrm{RT}}(A)$, where $\left|\partial V_{A}\right|$ denotes the number of edges that leaves $V_{A}$. Likewise, the $\zeta^{-1}$ domain $V_{B}$ is a cut for $B$ and the identity domain $V_{C}$ is a cut for $C$, so that $N\left|\partial V_{B}\right| \geq S_{\mathrm{RT}}(B)$ and $N\left|\partial V_{C}\right| \geq S_{\mathrm{RT}}(C)$. For each edge leaving $V_{A}$, the energy cost is at least 1 , and it is 2 if the edge enters one of the domains $V_{B}$ or $V_{C}$ (since $1, \zeta, \zeta^{-1}$ are even permutations). Thus the energy cost of an arbitrary configuration $\left\{s_{x}\right\}$ can be lower bounded by $N E\left[\left\{s_{x}\right\}\right] \geq S_{\mathrm{RT}}(A)+S_{\mathrm{RT}}(B)+S_{\mathrm{RT}}(C)$, with equality if and only if all three domains $V_{A}, V_{B}$, and $V_{C}$ are disjoint minimal cuts and if each connected component of the remaining bulk vertices is assigned a transposition. This can always be achieved, so

$$
E_{0}=\left[S_{\mathrm{RT}}(A)+S_{\mathrm{RT}}(B)+S_{\mathrm{RT}}(C)\right] / N,
$$

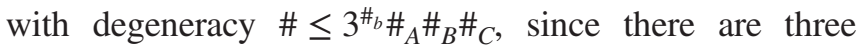
possible transpositions to choose from for each component (Fig. 2. right). If we combine these estimates with Eq. (5) and use some basic results for the trace, we obtain theorem 1 for qubits.

For $p \neq 2$, the stabilizer states no longer form a projective 3-design. To generalize our preceding argument, we derive a new formula for the third moment of a random stabilizer state $|V\rangle$ in $\left(\mathbb{C}^{p}\right)^{\otimes n}$, where $p \equiv 2(\bmod 3)$ and $n \geq 3$ ([37]):

$$
\langle\mid V\rangle\left\langle\left. V\right|^{\otimes 3}\right\rangle=\frac{1}{p^{n}\left(p^{n}+1\right)\left(p^{n}+p\right)} \sum_{T} R(T) .
$$

The sum is over the group $G_{3}(p)$ of orthogonal and doubly stochastic $3 \times 3$ matrices with entries in $\mathbb{F}_{p} ; R(T)$ is the corresponding operator defined on $\left(\mathbb{C}^{p}\right)^{\otimes 3 n}$ by $R(T)=r(T)^{\otimes n}, r(T)|\vec{q}\rangle=|T \vec{q}\rangle$ for $\vec{q} \in \mathbb{F}_{p}^{3}$. For qubits, $G_{3}(p)$ is equal to the permutation group; in general, it contains the latter as a proper subgroup. In contrast to previous results, which compute the frame potential of stabilizer states [31-33], our formula can be used to evaluate arbitrary third moments; we expect that Eq. (7) will be of independent interest in quantum information theory.

Just like in the case of qubits, the operators $R(T)$ act as a tensor product with respect to the $n$ copies of the singleparticle replica Hilbert space $\left(\mathbb{C}^{p}\right)^{\otimes 3}$. This is the central property that allows us to adapt the argument given above for qubits to obtain a classical ferromagnetic spin model with $G_{3}(p)$ degrees of freedom. Theorem 1 follows as above by an analysis of the low-temperature behavior of this model. See Ref. [37] for the technical details.

Discussion and outlook.-We have initiated a comprehensive study of multipartite entanglement in tensor network models of holography. Our results suggest several avenues for further investigation: First, it would be of mathematical interest to extend our analysis and establish sharp deviation bounds as in Ref. [9]. Second, tensor networks can also be used to define bulk-boundary mappings, or "holographic codes" $[18,19,28]$. In this case, the entanglement entropies of code states obtain a bulk correction, in agreement with the expectations of AdS/CFT [45], and it is natural to ask in which way the multipartite entanglement of typical code states is determined by the bulk [46]. Third, diagnostics such as moments of the partial transpose considered in this Letter may provide a path towards generalizing our results to nonstabilizer states and lead to a more refined understanding of multipartite entanglement in the AdS/CFT correspondence.

Random tensor networks have been a crucial source of inspiration for recent developments in information theory of quantum gravity, in part, due to complete analytical control over their bipartite entanglement structure. Some important examples include entanglement wedge reconstruction [47] and the recent progress on understanding the black hole information paradox [48]. In some cases, the connections go beyond mere inspiration, for instance, fixed-area states in quantum gravity mimic the entanglement properties of random tensor networks [49]. The stabilizer model presented in this Letter-while sharing the bipartite entanglement structure of random tensor networks-puts multipartite entanglement within reach of analytical tools.

It is a pleasure to thank David Gross, Patrick Hayden, Debbie Leung, Xiao-Liang Qi, Lenny Susskind, Zhao Yang, and Huangjun Zhu for inspiring discussions. S. N. acknowledges support from a Stanford Graduate Fellowship. M. W. gratefully acknowledges support from FQXI, the Simons Foundation, the DoD Multidisciplinary University Research Initiative (MURI), and an NWO Veni grant (No. 680-47-459).

[1] M. Walter, D. Gross, and J. Eisert, in Quantum Information: From Foundations to Quantum Technology Applications, 
2nd ed., edited by D. Bruss and G. Leuchs (John Wiley \& Sons, New York, 2019), p. 293.

[2] D. Gottesman, Ph.D. Thesis, Caltech, 1996.

[3] S. Bravyi, D. Fattal, and D. Gottesman, J. Math. Phys. (N.Y.) 47, 062106 (2006).

[4] S. Y. Looi and R. B. Griffiths, Phys. Rev. A 84, 052306 (2011).

[5] M. Hein, J. Eisert, and H.-J. Briegel, Phys. Rev. A 69, 062311 (2004).

[6] M. Van den Nest, J. Dehaene, and B. De Moor, Phys. Rev. A 71, 022310 (2005).

[7] M. Van den Nest, J. Dehaene, and B. De Moor, Phys. Rev. A 71, 062323 (2005).

[8] M. Hein, W. Dür, J. Eisert, R. Raussendorf, M. Nest, and H.-J. Briegel, in Quantum Computers, Algorithms and Chaos, Proceedings of the International School of Physics "Enrico Fermi" Vol. 162 (IOP Press, 2006), pp. 115-218, http://ebooks.iospress.nl/publication/27431.

[9] G. Smith and D. Leung, Phys. Rev. A 74, 062314 (2006).

[10] O. Dahlsten and M. B. Plenio, Quantum Inf. Comput. 6, 527 (2006).

[11] A. D. K. Plato, O. C. Dahlsten, and M. B. Plenio, Phys. Rev. A 78, 042332 (2008).

[12] B. Swingle, arXiv:1209.3304.

[13] B. Swingle, Phys. Rev. D 86, 065007 (2012).

[14] T. Hartman and J. Maldacena, J. High Energy Phys. 05 (2013) 014.

[15] S. Ryu and T. Takayanagi, Phys. Rev. Lett. 96, 181602 (2006).

[16] A. Lewkowycz and J. Maldacena, J. High Energy Phys. 08 (2013) 090.

[17] R. Orús, Ann. Phys. (Amsterdam) 349, 117 (2014).

[18] F. Pastawski, B. Yoshida, D. Harlow, and J. Preskill, J. High Energy Phys. 06 (2015) 149.

[19] Z. Yang, P. Hayden, and X.-L. Qi, J. High Energy Phys. 01 (2016) 175.

[20] P. Hayden, S. Nezami, X.-L. Qi, N. Thomas, M. Walter, and Z. Yang, J. High Energy Phys. 11 (2016) 009.

[21] M. B. Hastings, Commun. Math. Phys. 351, 387 (2017).

[22] P. Hayden, M. Headrick, and A. Maloney, Phys. Rev. D 87, 046003 (2013).

[23] V. Balasubramanian, P. Hayden, A. Maloney, D. Marolf, and S.F. Ross, Classical Quantum Gravity 31, 185015 (2014).

[24] D. Marolf, H. Maxfield, A. Peach, and S. Ross, Classical Quantum Gravity 32, 215006 (2015).

[25] L. Susskind, Fortschr. Phys. 64, 72 (2016).

[26] L. Susskind, Fortschr. Phys. 64, 551 (2016).

[27] D. Gross and M. Walter, J. Math. Phys. (N.Y.) 54, 082201 (2013).
[28] N. Bao, S. Nezami, H. Ooguri, B. Stoica, J. Sully, and M. Walter, J. High Energy Phys. 09 (2015) 130.

[29] N. Bao, C. Cao, M. Walter, and Z. Wang, J. High Energy Phys. 09 (2015) 203.

[30] D. M. Greenberger, M. A. Horne, and A. Zeilinger, in Bell's Theorem, Quantum Theory and Conceptions of the Universe (Springer, New York, 1989), pp. 69-72.

[31] R. Kueng and D. Gross, arXiv:1510.02767.

[32] H. Zhu, Phys. Rev. A 96, 062336 (2017).

[33] Z. Webb, Quantum Inf. Comput. 16, 1379 (2016).

[34] G. Evenbly and G. Vidal, J. Stat. Phys. 145, 891 (2011).

[35] A. Klappenecker and M. Rotteler, in Mutually Unbiased Bases are Complex Projective 2-Designs (IEEE, New York, 2005), pp. 1740-1744.

[36] D. Gross, K. Audenaert, and J. Eisert, J. Math. Phys. (N.Y.) 48, 052104 (2007).

[37] See Supplemental Material at http://link.aps.org/supplemental/ 10.1103/PhysRevLett.125.241602 for technical details, which includes Ref. [38].

[38] D. Gross, J. Math. Phys. (N.Y.) 47, 122107 (2006).

[39] In the language of Ref. [18], $\#_{b}$ is the number of multipartite residual regions.

[40] Note that $S(A)+S(B)+S(C)=2(a+b+c)+3 g$. It follows that if the sum of local entropies is odd then, necessarily, $g>0$. This is all that can be said about the tripartite entanglement from the knowledge of the entropies alone, and it justifies that the upper bound in Theorem 1 is never smaller than $\log _{p}(p+1) \geq 1$, even when the minimal cuts are unique.

[41] M. Christandl and A. Winter, J. Math. Phys. (N.Y.) 45, 829 (2004).

[42] J. Haah, Commun. Math. Phys. 324, 351 (2013).

[43] G. Salton, B. Swingle, and M. Walter, Phys. Rev. D 95, 105007 (2017).

[44] Note that $\operatorname{tr} \rho_{A B}^{T_{B}}=1$, and $\operatorname{tr}\left(\rho_{A B}^{T_{B}}\right)^{2}$ is the purity of system $A$, so it is an entropic measure. The third moment is the smallest moment containing nontrivial information about the multipartite entanglement.

[45] T. Faulkner, A. Lewkowycz, and J. Maldacena, J. High Energy Phys. 11 (2013) 074.

[46] In light of our approach, it is natural to conjecture that $S\left(\Phi_{V_{A}}\right)+S\left(\Phi_{V_{B}}\right)+S\left(\Phi_{V_{C}}\right)+\log _{p} \operatorname{tr}\left[\Phi^{\otimes 3} R_{V_{A}}(\zeta) R_{V_{B}}\left(\zeta^{-1}\right)\right.$ $R_{V^{\prime}}(\tau)$ ], where $\Phi$ is the inserted bulk state, will play a significant role.

[47] X. Dong, D. Harlow, and A. C. Wall, Phys. Rev. Lett. 117, 021601 (2016).

[48] G. Penington, J. High Energy Phys. 09 (2020) 002.

[49] X. Dong, D. Harlow, and D Marolf, J. High Energy Phys. 10 (2019) 240. 